\title{
On Investigating EMC Filter Solutions in Soft Open Points Under Large Unbalanced Current
}

\author{
Weichi Zhang \\ School of Engineering \\ Newcastle University \\ Newcastle upon Tyne, UK \\ weichi.zhang@ncl.ac.uk
}

\author{
Mohamed Dahidah \\ School of Engineering \\ Newcastle University \\ Newcastle upon Tyne, UK \\ mohamed.dahidah@ncl.ac.uk
}

\author{
Graeme Thompson \\ Turbo Power Systems \\ Gateshead, UK \\ Gthompson@turbopowersystems.c \\ om
}

\author{
Volker Pickert \\ School of Engineering \\ Newcastle University \\ Newcastle upon Tyne, UK \\ Volker.pickert@ncl.ac.uk
}

\author{
Mohammed Elgendy \\ School of Engineering \\ Newcastle University \\ Newcastle upon Tyne, UK \\ Mohammed.elgendy@ncl.ac.uk
}

\begin{abstract}
An emerging network enhancement, Soft Open Points (SOPs), has been investigated to bring flexibility to the overall Low-Voltage (LV) network. The utilization of Power Electronics Devices (PEDs) in the form of SOP accommodates the increases in load and connected distributed generation (DG), meanwhile supporting grid operations with supplementary functionalities. However, owing to its designated operation and unique topology, there are challenging issues in its practical implementation. This paper investigates the ever-challenging Electro-magnetic Compatibility (EMC) issues resulted from the unbalanced operation of SOP. Two novel approaches have been proposed to tackle the issue. The feasibility and performance of the proposed solutions are verified through mathematical derivations and extensive simulation studies.
\end{abstract}

Keywords - LV network enhancement, Soft Open Points, CM core, Unbalanced Current, EMC

\section{INTRODUCTION}

With the increasing attention on Low Carbon Technologies (LCT), the widespread use of DG, energy storage as well as the introduction of controllable loads and electric vehicles have been connected to the LV networks. The accumulative effect from these different operational regimes can lead to problems in the operation of distribution networks such as undesirable voltage excursions and feeder load unbalance, whereas mitigating these issues with traditional methods remains a technical challenge. New design and configurations must be adopted in the distribution network to facilitate a more efficient connection of increased LCT, meanwhile providing power flow management and voltage regulation when infrastructure upgrades or active compensation are required [1]. Without changing network topology, an alternative technology - Soft Open Points (SOPs), has been investigated in the literature to tackle the abovementioned challenges [2-4].

SOPs are power electronic devices installed in place of normally open points that connect adjacent feeders in a distribution network to provide alternative routes of electricity supply. The multi-terminal PEDs are connected to a common internal DC link as a Back-to-Back voltage-source converter (VSC), exchanging power between terminals thus between LV network substations. The use of multi-terminal PED in the form of SOP facilitates the support of smart LV network functionality and the controllable power transfer between neighbouring feeders. In addition, as both current and voltage can be manipulated in each individual phase, it can also perform other supplementary functionalities such as phase imbalance improvement, voltage support, power quality improvement. Therefore, the implementation of SOP brings flexibility to the overall LV network and improves the utilisation of the existing infrastructure $[3,5]$. Although the benefits of SOP for distribution network operation are quite obvious, however, there are still technical challenges regarding its practical implementation. Particularly, the Electro-magnetic interference (EMI) emission from the PEDs terminals remains a potential concern.

As all electrical and electronic apparatus have to show compliance to essential EMC emission requirements such as CISPR 16 before they can be connected to the distribution network [6], therefore, dedicated EMC filters are usually placed at the output before the connection point. While the updated SOP system utilizes the-state-of-the-art Silicon Carbide ( $\mathrm{SiC}$ ) semiconductor technology to minimize the switching losses and the size of output magnetic circuit, the increased $d v / d t$ is accompanied by the increase of the EMI level. Even with adopting some EMI mitigation techniques [7], dedicated filtering is still required to satisfy the strict EMI standard and grid compliance. However, due to the multiterminal topology and designated load-balancing requirements, the phase current output from the individual PED terminal could be unbalanced. Particularly during some grid fault conditions, the unbalanced current can reach as high as its rated current. As a result, these significantly increase the complexity of EMC filter design.

Fig. 1 shows a dual-terminal (2T) PEDs SOP that consists of two three-phase VSCs and a single-phase converter. The neutral and earth connections are joined together to the midpoint of the DC bus. The single-phase converter is implemented for the purpose of neutral point control, actively improving the performance of the split DC topology [8-10]. As previously mentioned, the phase voltage and current in three-phase VSC can be controlled independently. This facilitates power exchange between individual phases, thus avoiding overload of feeders and transformers. Combined with the neutral point control, these output ports form a 7phase topology (this is more complicated in 10-phase tripleterminal PEDs system) where the phase current can potentially be imported or exported between different substation networks and phases. This results in high unbalanced output at each terminal even though the overall power exchange is still balanced within the SOP. Due to the high current rating and large power managing capability in 


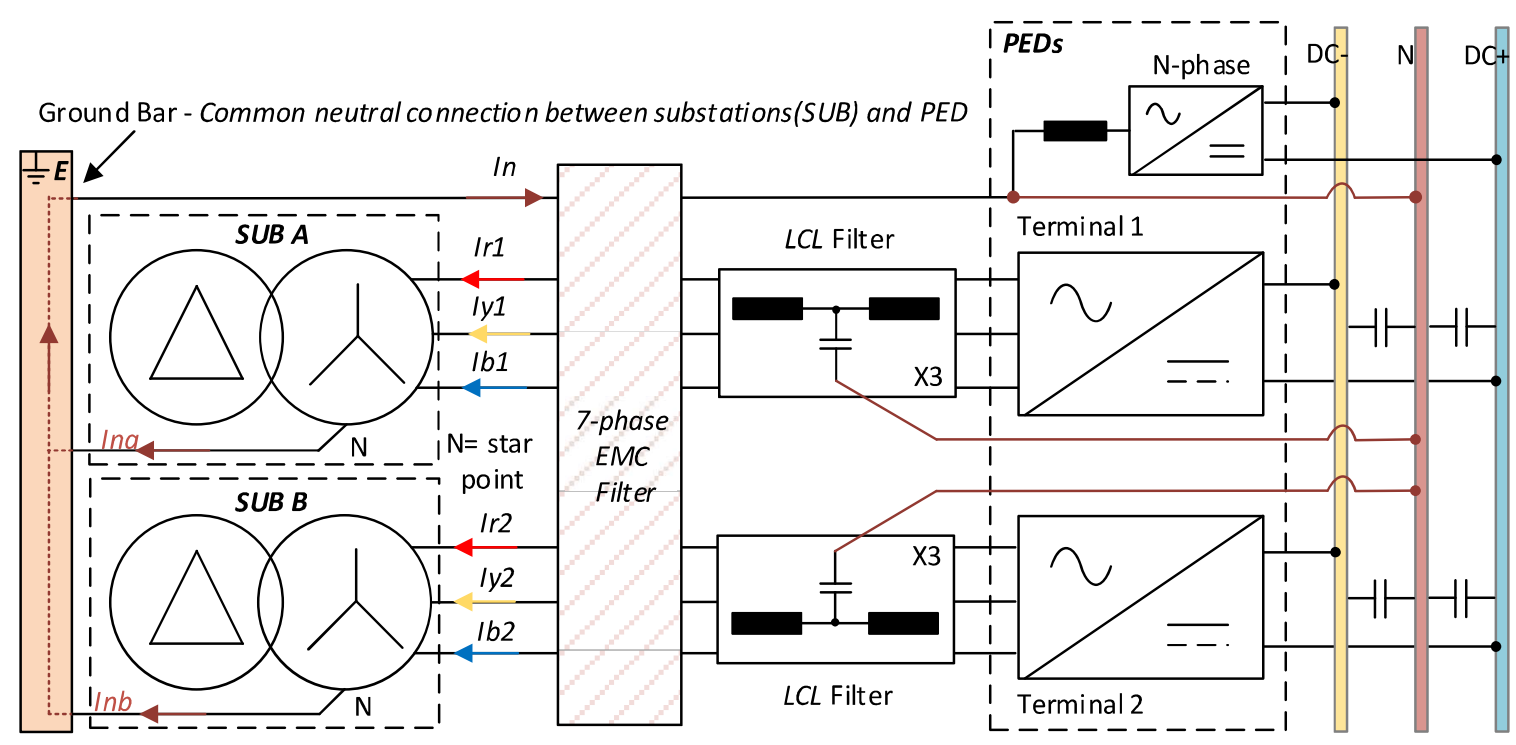

Fig. 1. Diagram of the dual-terminal (2T) PEDs in the form of SOP with a 7-phase EMC filter

these VSCs (two $240 \mathrm{kVA}$ in a dual-terminal PEDs and 400 $\mathrm{kVA}$ in the case of a triple-terminal PEDs)[9], the unbalance issue at each terminal poses a challenging EMC filter implementation. Since EMC filter is typically composed of common-mode $(\mathrm{CM})$ cores and bypass $\mathrm{X}$ or $\mathrm{Y}$ capacitors, the remaining flux generated by the large unbalanced current can saturate these CM cores, thus passing the attenuation solely on the filter capacitors. This significantly reduces the overall CM noise rejection as well as the lifetime of filter capacitors.

While the overall power and phase current is still balanced within the multi-terminal PEDs, minimal net flux generated from differential phase current can be achieved by passing all phases through the CM core, as shown in Fig. 1. However, this is not a cost-effective solution for SOP application as its rated current per phase is above $300 \mathrm{~A}$. Not only huge filter cores are needed to pass all thick wires, owing to space constraint; but very limited CM attenuation is obtained from this giant filter configuration. Therefore, optimal EMC filter solutions in SOP applications should carefully consider optimizing the volume, weight as well as cost-effectiveness.

Apart from the conventional LC structure, many studies have investigated EMC filter design from different perspectives. For example, active filters are utilized in addition to the LC passive filter to reduce its size and cost [7, 11], however, they are also prone to unbalance issues. Whilst some other research has looked into unbalanced issues caused by filter parameters or noise path $[13,14]$, they cannot fit the SOPs as of which the unbalance current is intrinsically large during operation.

In this paper, two novel core compensation approaches are investigated to cancel the magnetic flux generated from the large unbalanced current. The techniques prevent the CM core from saturation with an unbalanced current up to $350 \mathrm{~A}$. This facilitates the EMC filter design at individual PED terminal, resulting in a reduction of the cost and size of EMC filters. The remainder of the paper is structured as follows: Section II illustrates the proposed passive compensation solution with mathematical models. Design precautions are also highlighted in practical implementation, and an alternative modification, dual-stage filtering solution, is introduced. Section III reports the results of conventional EMC filter and the two proposed solutions based on an unbalanced PED model. Finally, some conclusions are summarized in Section V.

\section{LINE-FREQUENCY FLUX CANCELLATION}

In general, conducted noises generated in a converter are classified into CM and differential mode (DM). The DM noise is mostly at switching frequency or its integer harmonics, which is normally tackled by the output PWM filter. Whereas the CM current flowing through parasitic capacitance in the power circuit causes most of EMI issues. In other words, EMI emission can be greatly suppressed by mitigating the CM noise. Since the CM current is conducted along the phases in the same direction and in phase, in a typical EMI filter, the $\mathrm{CM}$ current generates an accumulated magnetic flux on the filter cores whilst the DM current flowing through causes an opposite magnetic flux and a theoretical zero net flux. As a result, the cores produce a high impedance on $\mathrm{CM}$ current with minimal impact on the DM phase current. However, due to the unbalanced issue associated with the SOPs operation, the unbalanced phase current generates a large flux at $50 \mathrm{~Hz}$, which saturates the CM cores in the EMI filter. The change of core inductance can be seen from (1), where a significant reduction of $\mathrm{CM}$ attenuation is found during saturation when its permeability is minimal $\left(\mu_{\text {sat }} \ll \mu\right)$ :

$$
L_{C M}=N^{2} \cdot \frac{\mu A_{e}}{l_{e}}
$$

where $\mathrm{N}$ is the number of turns on the core, $A_{e}$ is the effective cross-sectional area and $l_{e}$ is the effective length of the magnetic path of the core. Although manipulating these core parameters can counter the saturation in some extent, this still decreases the core inductance thus attenuation on CM current, and a larger size is needed for large unbalanced current.

Alternatively, instead of increasing the core size, the CM core inductance can be restored through re-balancing the linefrequency DM current therefore flux. In this manner, the remaining high-frequency flux generated by $\mathrm{CM}$ current can accumulate on the core, providing $\mathrm{CM}$ current rejection without saturation. This paper introduces different approaches to compensate for the flux generated from the phase current as discussed in the following subsections. 


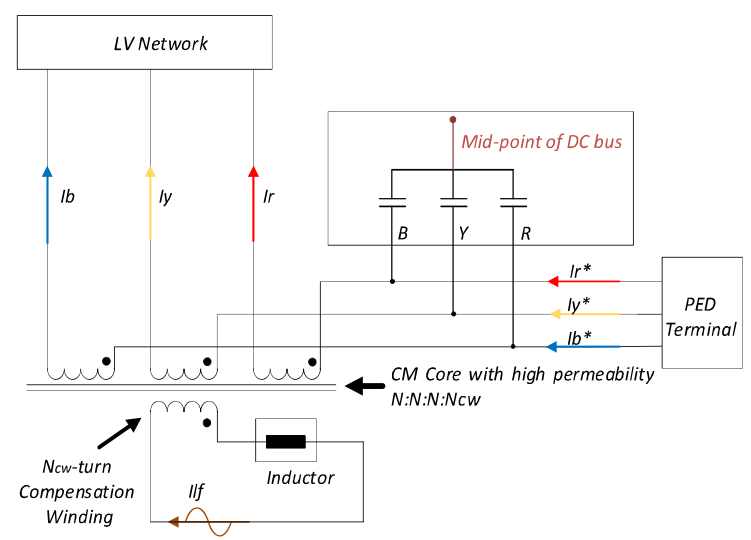

Fig. 2. Prposed core compensation under large unbalanced current

\section{A. Passive Core Compensation with Inductor}

Fig. 2 illustrates a simple passive compensation solution using a multi-turn winding on the filter core in series with a dedicate inductor. This operates the filter core as a current transformer (CT) where the saturation under large unbalanced current is compensated by the magnetizing current flowing through the secondary compensation winding. However, the high-frequency current induced from CM current will cancel out its magnetic flux thus the $\mathrm{CM}$ attenuation. To preserve the core attenuation, a dedicated inductor is connected to the compensation winding, removing the highest possible highfrequency magnetizing current on the secondary. In addition, bypass capacitors are placed before the cores to provide low impedance paths for CM current circulating to the mid-point of the DC bus/Protective earth (PE).

As shown in Fig. 2, $I_{b}^{*}, I_{y}^{*}, I_{r}^{*}$ are the currents from a single PED terminal which are fed into the proposed core compensation solution, and they could be significantly unbalanced. The high-permeability filter cores are winded with a $N_{c w}$-turn compensation winding on the secondary. In this manner, the core works as a four-winding $\mathrm{CT}$ with a ratio of $N: N: N: N_{c w}$. With the proposed structure, the CM current should be greatly suppressed at the grid-side output, thus the EMI emission. This is further proved by the mathematical analysis below.

\section{B. Mathematical Analysis and Models}

The magnetic field of CM core is induced by the current flowing through the windings, as follows:

$$
H_{C M C}=\frac{N i}{l_{e}}=\frac{N i_{r}}{l_{e}}+\frac{N i_{y}}{l_{e}}+\frac{N i_{b}}{l_{e}}
$$

where $N$ is the number of turns on each phase and $l_{e}$ is the effective length of the core magnetic path. $i_{r}, i_{y}, i_{b}$ are the phase current $\left(i_{i}\right)$ that can also be represented according to the conduction mode, as:

$$
\begin{gathered}
i=i_{r, D M}+i_{y, D M}+i_{b, D M}+i_{r, C M}+i_{y, C M}+i_{b, C M} \\
=i_{i, D M}+i_{i, C M}
\end{gathered}
$$

From (2) and (3), yield:

$$
H_{C M C}=H_{C M C, D M}+H_{C M C, C M}=\frac{N\left(i_{i, D M}+i_{i, C M}\right)}{l_{e}}
$$

In the case of unbalanced SOP operation $\left(i_{i, D M} \gg 0\right)$, the magnetic field $H_{C M C, D M}$ generated from large unbalanced current saturates the core, thus significantly decreasing the core impedance on CM currents. This have been seen in (1), where $L_{C M}=0$.

However, by including the compensation winding circuit, the large DM magnetic field is cancelled out in the same manner as a CT. The line-frequency magnetizing current $i_{l f}$ induced by the unbalanced phase current is determined as:

$$
i_{l f}=-\frac{N\left(i_{r, D M}+i_{y, D M}+i_{b, D M}\right)}{N_{c w}}=-\frac{N i_{i, D M}}{N_{c w}}
$$

Where, $N_{C w}$ represents the number of turns of the compensation winding, and the DM net flux of the core, which is given as:

$$
H_{C M C, D M}=\frac{N i_{i, D M}}{l_{e}}+\frac{N_{c w} i_{l f}}{l_{e}}=0
$$

Besides the magnetizing current at line-frequency, due to the operation of CT, there are also high-frequency current $i_{h f}$ induced from the $\mathrm{CM}$ current, where:

$$
i_{h f}=-\frac{N}{N_{c w}}\left(i_{r, C M}+i_{y, C M}+i_{b, C M}\right)=-\frac{N}{N_{c w}} i_{i, C M}
$$

This induced $i_{h f}$ will cancel out the magnetic field $H_{C M C, C M}$ on the core as well as the impedance to CM current. With including the compensation winding, three-phase CM core can be equivalently represented as a four-phase model:

$$
\left[\begin{array}{c}
V_{r} \\
V_{y} \\
V_{b} \\
V_{c w}
\end{array}\right]=\left[\begin{array}{cccc}
L & M & M & M_{c w} \\
M & L & M & M_{c w} \\
M & M & L & M_{c w} \\
M & M & M & L_{c w}
\end{array}\right] \cdot \frac{d}{d t}\left[\begin{array}{c}
i_{r} \\
i_{y} \\
i_{b} \\
i_{c w}
\end{array}\right]
$$

where $L$ and $M$ are the three-phase inductance and mutual inductance (assuming three-phase winding are identical, $L=$ $L_{r}=L_{y}=L_{b}$ ), respectively. $V$ and $i$ represent the induced back emf and magnetizing current on each phase as well as compensation winding. From (8), the voltage across the compensation winding $V_{c w}$ is calculated as:

$$
V_{c w}=M \frac{d i_{r}}{d t}+M \frac{d i_{y}}{d t}+M \frac{d i_{b}}{d t}+L_{c w} \frac{d i_{c w}}{d t}
$$

As portrayed in Fig. 2, an inductor in series with the compensation winding circuit and collaboration from bypass capacitors results in minimizing the induced high-frequency current, $i_{h f}$. Consequently, the induced current $i_{c w}$ flowing through compensation winding contains the minimal highfrequency component; hence the approach presents high $\mathrm{CM}$ attenuation. Furthermore, assuming a large inductor is used, the compensation winding circuit can be seen as an equivalent open circuit at high frequency, and its CM current and voltage can be neglected $\left(i_{c w, C M}, V_{c w, C M}=0\right)$. Whilst for DM current at line-frequency, the $\mathrm{CM}$ core operates as a $\mathrm{CT}$ and the compensation winding is an equivalent short circuit. In this manner, the ideal CM and DM models of the filter core with proposed compensation are achieved in Fig. 3 and Fig. 4, respectively.

Since it operates as three coupled inductors at CM, (8) can be simplified as (10), regardless of its large unbalance DM current:

$$
\left[\begin{array}{l}
V_{r, C M} \\
V_{y, C M} \\
V_{b, C M}
\end{array}\right]=\left[\begin{array}{ccc}
L & M & M \\
M & L & M \\
M & M & L
\end{array}\right] \cdot \frac{d}{d t}\left[\begin{array}{l}
i_{r, C M} \\
i_{y, C M} \\
i_{b, C M}
\end{array}\right]
$$




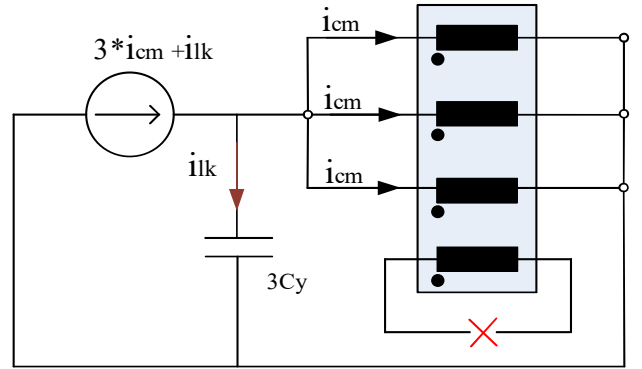

Fig. 3. CM model of filter core with compensation winding

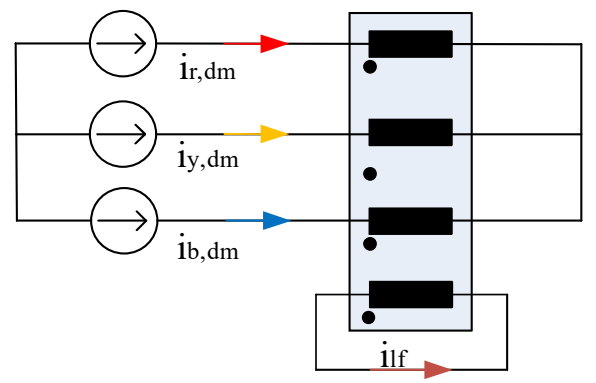

Fig. 4. DM model of filter core with compensation winding

Assuming the identical $\mathrm{CM}$ current $\left(i_{r, C M}=i_{y, C M}=\right.$ $\left.i_{b, C M}\right)$ flowing through the phase winding with a coupling factor $k_{m}=1$, based on the model in Fig . 4, the effective CM inductance $L_{r-e f, C M}$ can then be calculated from (10):

$$
\begin{gathered}
L_{r-e f, C M}=\frac{V_{r, C M}}{s i_{r, C M}} \\
=\frac{s\left(L i_{r, C M}+M i_{y, C M}+M i_{b, C M}\right)}{s i_{r, C M}}=L+M+M
\end{gathered}
$$

Whilst effective DM inductance of the CM core can be derived from (8):

$$
L_{r-e f, D M}=\frac{V_{r, D M}}{s i_{r, D M}}=\frac{L i_{i, D M}+M_{c w} i_{c w, D M}}{i_{r, D M}}=0
$$

Consequently, proposed passive compensation solution re-balances the large line-frequency flux, thus avoiding core saturation. The calculation shows effective CM inductance to suppress the EMI emission under large unbalanced current, meanwhile having minimal impact on the phase current. In addition, the proposed EMC filter is not only limited to threephase output, the compensation winding always balances the flux regardless of the phase configuration, therefore it fits well with the SOPs requirements.

It worth mentioning that high-permeability core materials can be used, and from (1), the core size and turns need to be optimized to achieve the highest self-inductance $L$ possible [16], as:

$$
L(f)=N^{2} A_{L} \frac{\mu(f)}{\mu(10 k H z)}
$$

where, $A_{L}$ is the inductance coefficient given by the manufactures, which represents the inductance value per turn at certain frequencies (normally at $10 \mathrm{kHz}$ or $100 \mathrm{kHz}$ ).

\section{Practical Design Criteria of Series Inductor}

Although theoretical derivations have proven the viability of the ideal line-frequency flux cancellation using proposed

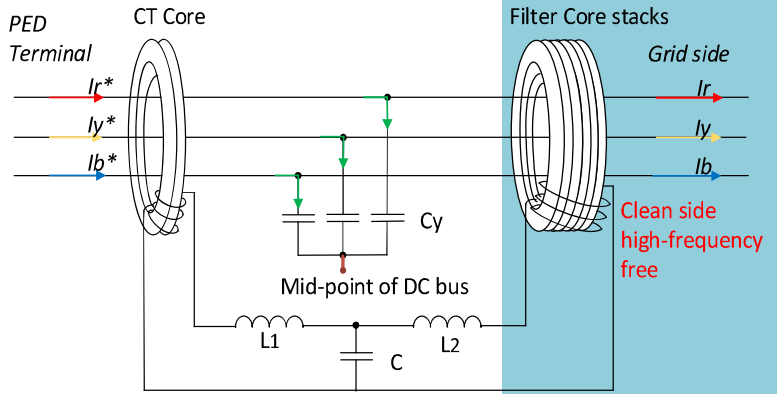

Fig. 5. Dual-stage modification connected to PED terminal output

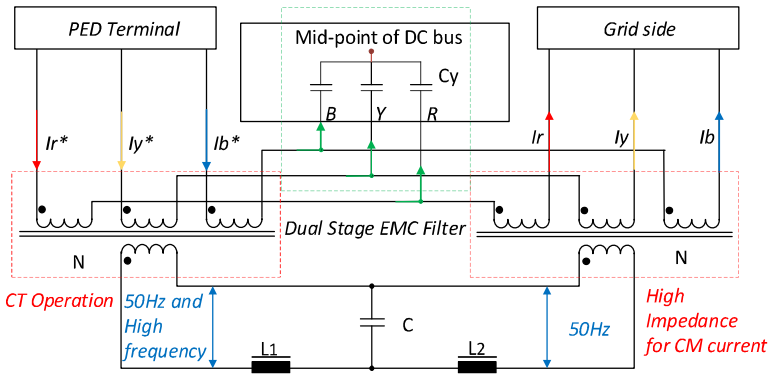

Fig. 6. Schematic diagram of dual-stage modification

compensation, however, in practical implementation, the series-connected inductor on the compensation circuit must be carefully designed to preserve the highest $\mathrm{CM}$ attenuation possible. Eq. (14) shows the core CM impedance $Z_{C M}$ with respect to the number of turns $N_{c w}$ and the series inductor $L_{s}$ :

$$
Z_{C M}=\frac{j \omega L_{s}}{N_{c w}^{2}}
$$

To achieve the same core impedance, the more turns on the compensation winding, the higher the inductance required. For example, obtaining $50 \mathrm{~dB}$ attenuation at $1 \mathrm{MHz}$ with a 15-turn compensation winding requires a series inductance of $0.8 \mathrm{mH}$. Nevertheless, the impedance of compensation circuit at line-frequency must be taken into consideration when selecting the number of turns $N_{c w}$ as the cores are incapable of carrying high VA burden. With high secondary impedance or fewer turns, the filter cores could still be saturated at $50 \mathrm{~Hz}$ even by adopting the proposed compensation technique.

Lastly, the stray capacitance of the inductor has to be taken into consideration as it provides potential conducting paths for high-frequency current. To minimize this capacitive effect on attenuation, the inductor needs to be designed with a high selfresonant frequency (SRF) at $\mathrm{MHz}$, this would significantly increase the design complexity and cost of the series inductor.

It is worth concluding that the proposed compensation can balance the core flux at $50 \mathrm{~Hz}$ and hence avoid the saturation, but the $\mathrm{CM}$ attenuation is constrained by the inductor design. To lessen the design complexity and requirements, the above passive compensation is modified into a dual-stage solution.

\section{Dual-Stage Modification}

Based on the proposed line-frequency flux cancellation, the diagram of dual-stage modification is shown in Fig. 5. The filter cores are split into two separate stages, where their secondary is winded with the same turns and connected via an $L C L$ filter. The front stage of this configuration is utilised as a $\mathrm{CT}$ for current sensing, whereas the second stage carries out 
TABLE I. SimULATION PARAMETERS

\begin{tabular}{ccc}
\hline \hline Symbol & Quantity & Value \\
\hline$I_{u n}$ & PED unbalanced current & $325 \mathrm{~A}$ \\
$A_{L}$ & W587-Inductance coefficient at $100 \mathrm{kHz}$ & $14.8 \mu \mathrm{H}$ \\
$L_{s}$ & Series Inductor & $0.8 \mathrm{mH}$ \\
$C_{y}$ & Bypass Caps in dual-stage solution & $1 \mu \mathrm{F}$ \\
$N_{c w}$ & Turns ratio of compensation winding & 15 \\
$f_{s r}$ & SRF of series inductor & $1.6 \mathrm{MHz}$ \\
$f_{l c l s r}$ & SRF of inductors in $L C L$ & $5 \mathrm{MHz}$ \\
\hline
\end{tabular}

the filtering duties. The operation is further illustrated in Fig. 6 , where capacitors are in place between two stages, providing a relative low impedance path for CM currents. Furthermore, substituting the series inductor with an $L C L$ reduces the size and design complexity, releasing the VI burden of the cores. From this configuration, the compensation circuit offers 60 $\mathrm{dB} /$ decade attenuation above its cut-off frequency, thus only unbalanced phase current flows to the second stage and rebalances the saturation of the filtering cores. Consequently, the dual-stage structure provides high $\mathrm{CM}$ attenuation on the output where the most CM noise currents are shunted from phase current to the mid-point of DC via bypass capacitors.

The cores can vary from two stages as the in front stage mainly deals with the line-frequency current sensing, in which the core can be less frequency dependent. Whereas the second stage provides $\mathrm{CM}$ attenuation, thus requiring the cores with a higher permeability. It worth highlighting that for the same front stage setup, multiple cores can be stacked in the second stage to boost the CM attenuation as illustrated in Fig. 5 .

\section{Simulation Results}

Simulation models are developed using PLECS software package to validate the proposed solutions. As the aim of this paper is to evaluate the performance of the EMC filter in SOPs under large unbalanced current, each individual phase module of PED is modelled as a separate voltage source. Based on the design specifications of 2T SOP, an unbalanced phase current of $325 \mathrm{~A}$ is introduced to the PED model which imitates the extreme operating condition. The proposed filtering solutions are connected between the output of individual PED terminal and the grid, with including a stack of five CM cores (VITROPERM L2130-W587) from Vacuumschmelze. The core is selected based on the design space envelope and wires thickness of the SOP system. Based on the datasheet, an equivalent magnetic model of filter cores is also established in each simulation model to monitor the core magnetization. Some simulation parameters are summarized in Table I. For testing purposes, $\mathrm{CM}$ noise currents at selected frequencies $(0.15,0.25,0.35,0.45 \mathrm{MHz})$ are manually injected to the simulation model based on the Quasi-peak level of lowvoltage AC mains port in CIRSP 16 (79 $d B$ from 0.15 to 0.5 $\mathrm{MHz}, 73 \mathrm{~dB} 0.5$ to $30 \mathrm{MHz}$ ) [6].

First, an EMC filter composed of stacked CM cores and bypass capacitors is connected to the unbalanced PED model without compensation. The magnetization curve (red) in Fig.7 shows that the cores are greatly saturated at $50 \mathrm{~Hz}$. As a result, all filtering burdens are solely relied on the capacitors. By introducing CM noise current to the simulation, FFTs of CM noise voltage across nominal $50-\Omega$ LISN impedance are

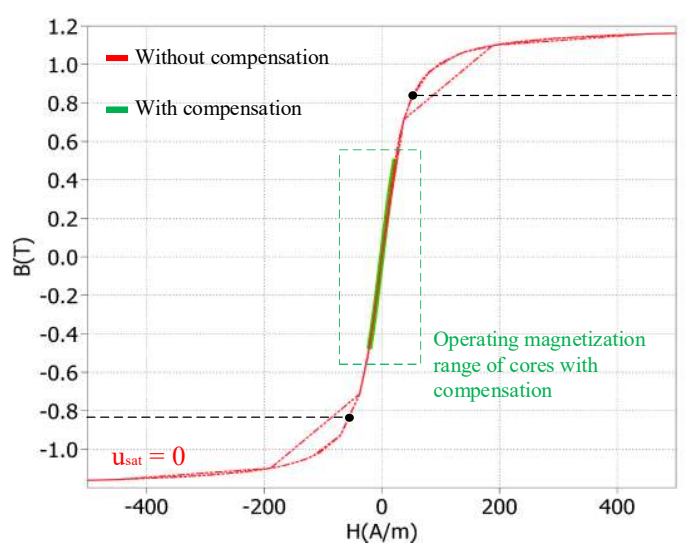

Fig. 7. Magnetization curve with/without passive compensation

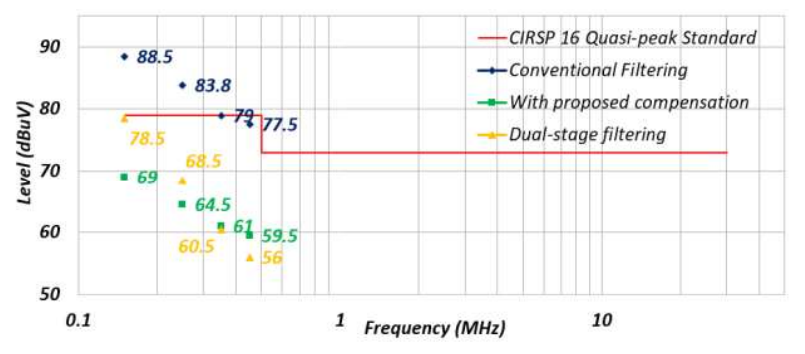

Fig. 8 Measurements of EMI emission level

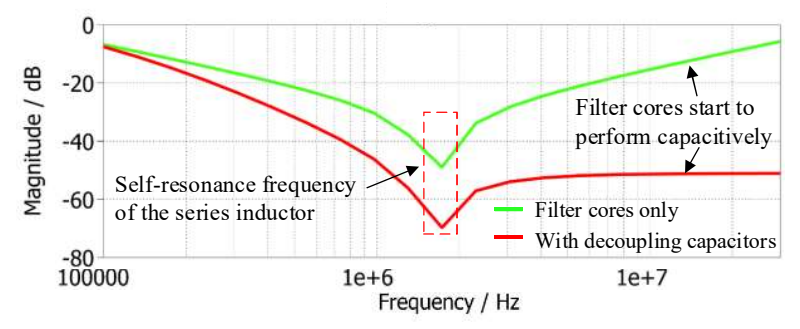

Fig. 9. Filter cores attenuation over frequency with compensation

shown in Fig. 8, where the EMI emission subject to this filter fails to comply with the CISPR standard.

Applying the proposed compensation approach in Fig. 2, where an inductor of $0.8 \mathrm{mH}$ is connected in series with an ESR of 0.1 on the secondary, the filter is tested again under the same unbalance condition. The magnetization curve of filter cores is shown in Fig. 7 (green), which indicates a linear magnetic operation. The EMI emission can also be observed from Fig. 8, where the CM noises are greatly suppressed with leaving sufficient margin to the standard. Nevertheless, Fig. 9 shows the cores attenuation and the corresponding attenuation with the bypass capacitors over frequency spectrum. It can be observed that even by adopting the proposed compensation, the core attenuation decreases after reaching the SRF of the series inductor at $1.6 \mathrm{MHz}$, whilst the overall $\mathrm{CM}$ attenuation of the filter stays at $-50 d B$, applied by the bypass capacitors.

The PED model is then simulated with the dual-stage structure as in Fig. 5, where the core used for CT operation is also selected as $W 587$ for simplicity. The passive components of $L C L$ filter are significantly smaller; a $10 \mu \mathrm{H}$ inductor at front stage of compensation circuit, a $100 \mu \mathrm{H}$ inductor on the latter stage and a $1 \mu F$ capacitor, forming a cut-off frequency of $52.8 \mathrm{kHz}$. To note that higher SRF ( $5 \mathrm{MHz}$ in simulation) is applicable to the inductor due to its smaller inductance. 


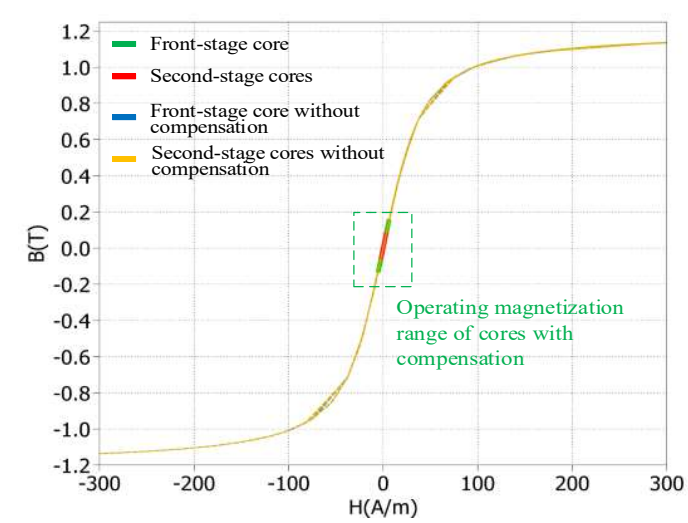

Fig. 10. Magnetization curve of dual-stage modification

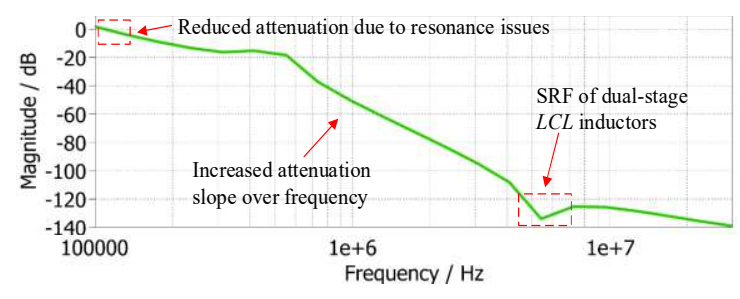

Fig. 11. Attenuation of dual-stage cores configuration over frequency

Fig. 10 shows the magnetization curve under the same unbalance current, where the cores in both stages operate within linear magnetization range through adopting the linefrequency cancellation. In addition, compared to Fig. 7, reduce in inductance on the compensation circuit reduces the VI burden of the core, therefore improving the core linear operating margins $( \pm 0.2 T)$ against saturation.

Fig. 11 shows the attenuation of dual-stage modification over the frequency spectrum. Compared with single inductor compensation, greater attenuation is attained with the increase of frequency. With improved inductor SRF, this configuration facilitates EMI mitigation at higher frequencies. However, the use of bypass capacitors, and secondary $L C L$ filter results in resonance issues at lower frequency, therefore affecting the mitigation performance. With injecting the same CM noise currents, the corresponding EMI emissions are also captured in yellow in Fig. 8. In this simulation, the EMI emission level from dual-stage filter setup barely passes $79 \mathrm{~dB}$ at $150 \mathrm{kHz}$, whereas it shows good compliance at higher frequencies. It worth noting that once the EMI emission fails to comply with the standard in some contingencies at low frequencies, the mitigation performance can be easily improved by tuning the resonance (e.g. increase the front stage inductance in $L C L$ or bypass capacitance), without saturating the cores in the filter.

\section{CONCLUSION}

With an increase of DG volumes, a viable innovative power management technique, SOPs, is emerging to adopt more effective and efficient intervention in the distribution network. This paper investigated the ever-challenging EMC issues in SOPs application for the LV network. Based on the proposed line-frequency flux cancellation, two novel solutions have been investigated in this paper, facilitating EMI mitigation at individual PED terminal with reduced size and cost. The mathematical derivations and models have proven the line-frequency flux cancellation in proposed solutions working under large unbalanced current. Through modelling PED and filter in PLECS, the core magnetization and filter performance have been validated from the extensive simulation results. While the concept viability has been verified, there still will be an ongoing investigation to optimize the filter design and its practical implementation to fit for any other heavily unbalanced applications.

\section{ACKNOWLEDGEMENT}

The multi-terminal PED has been developed within Turbo Power Systems (TPS) in collaboration with UK Power Networks and Imperial College London. This particular work is a Knowledge Transfer Partnership (KTP) project funded by Innovate UK, collaborated between TPS and Newcastle University.

\section{REFERENCES}

[1] J. M. Bloemink and T. C. Green, "Benefits of distribution-level power electronics for supporting distributed generation growth," IEEE Transactions on Power Delivery, vol. 28, no. 2, pp. 911-919, 2013.

[2] W. Cao, J. Wu, and N. Jenkins, "Feeder load balancing in MV distribution networks using soft normally-open points," in IEEE PES Innovative Smart Grid Technologies, Europe, 2014, pp. 1-6: IEEE.

[3] W. Cao, J. Wu, N. Jenkins, C. Wang, and T. Green, "Benefits analysis of Soft Open Points for electrical distribution network operation," Applied Energy, vol. 165, pp. 36-47, 2016.

[4] C. Wang, G. Song, P. Li, H. Ji, J. Zhao, and J. Wu, "Optimal siting and sizing of soft open points in active electrical distribution networks," Applied energy, vol. 189, pp. 301-309, 2017.

[5] W. Cao, J. Wu, N. Jenkins, C. Wang, and T. Green, "Operating principle of Soft Open Points for electrical distribution network operation," Applied Energy, vol. 164, pp. 245-257, 2016.

[6] I. CISPR, "16-1-2: Specification for radio disturbance and immunity measuring apparatus and methods-Part 1-2: Radio disturbance and immunity measuring apparatus-Ancillary equipment-Conducted disturbances," IEC, Geneva, 2006.

[7] M. R. Yazdani, H. Farzanehfard, and J. Faiz, "Classification and comparison of EMI mitigation techniques in switching power converters-A review," Journal of Power Electronics, vol. 11, no. 5, pp. 767-777, 2011.

[8] T. Hornik and Q.-C. Zhong, "Parallel PI voltage-H $\infty$ current controller for the neutral point of a three-phase inverter," IEEE Transactions on Industrial Electronics, vol. 60, no. 4, pp. 1335-1343, 2013.

[9] T.Hornik, D.Charlton, S.Mitchell, F.Hassan, N.Jakeman, I.Jonhnson, T.Lakin and P.Lang, "An active power management in LV distribution network by using the multi-terminal power electronics devices," in $7 \mathrm{th}$ IEEE PES Innovative Smart Grid Technologies Conference Europe (ISGT-Europe), Torino, Italy, 2017.

[10] J. Liang, T. C. Green, C. Feng, and G. Weiss, "Increasing voltage utilization in split-link, four-wire inverters," IEEE Transactions on Power Electronics, vol. 24, no. 6, pp. 1562-1569, 2009.

[11] Y.-C. Son and S.-K. Sul, "A new active common-mode EMI filter for PWM inverter," IEEE Transactions on Power Electronics, vol. 18, no. 6, pp. 1309-1314, 2003.

[12] J. Xue, F. Wang, and B. Guo, "EMI noise mode transformation due to propagation path unbalance in three-phase motor drive system and its implication to EMI filter design," in 2014 IEEE Applied Power Electronics Conference and Exposition-APEC 2014, 2014, pp. 806811: IEEE.

[13] D. Bellan, "Mode transformation of EMI noise due to unbalanced filter capacitors in three-phase motor drive systems," in IECON 2017-43rd Annual Conference of the IEEE Industrial Electronics Society, 2017, pp. 7024-7027: IEEE.

[14] M. L. Heldwein, L. Dalessandro, and J. W. Kolar, "The three-phase common-mode inductor: Modeling and design issues," IEEE Transactions on Industrial Electronics, vol. 58, no. 8, pp. 3264-3274, 2011. 